\title{
NÚMERO MÍNIMO DE ARMADILHAS DE MÖRICKE EM AMOSTRAGEM DE HIMENÓPTEROS PARASITÓIDES NA CULTURA DA SOJA GLYCINE MAX (L.) MERRILL
}

\section{R.I.R. Lara ${ }^{1}$, N.W. Perioto $^{1}$, Z.A. Ramiro ${ }^{2}$}

${ }^{1}$ Apta Regional Centro-Leste, Laboratório de Bioecologia e Taxonomia de Parasitóides e Predadores, Rua Peru, 1472-A, CEP 14075-310, Ribeirão Preto, SP, Brasil. E-mail: rirlara@apta.sp.gov.br

\section{RESUMO}

\begin{abstract}
O objetivo do presente estudo foi avaliar o número mínimo de armadilhas de Möricke para a amostragem da diversidade de himenópteros parasitóides na cultura da soja Glycine max (L.) Merrill. O experimento foi conduzido em cultivo de soja, da variedade IAC 82, em Ribeirão Preto ( $\left.21^{\circ} 12^{\prime} 17,8^{\prime \prime} \mathrm{S} / 47^{\circ} 52^{\prime} 34,8^{\prime \prime} \mathrm{O}\right), \mathrm{SP}$, Brasil. No período de 24 de fevereiro a 11 de abril de 2003 foram realizadas sete coletas de himenópteros parasitóides com armadilhas de Möricke. Utilizou-se o delineamento estatístico de blocos casualizados, com cinco tratamentos (uma a cinco armadilhas) e quatro repetições. Quanto ao total de famílias capturadas, as maiores médias foram obtidas com a utilização de duas a cinco armadilhas de Möricke, que diferiram significativamente da média obtida com uma armadilha. Estes resultados indicam que, para parcelas de $12 \times 18 \mathrm{~m}$, duas armadilhas de Möricke são suficientes para amostrar a diversidade de himenópteros parasitóides em cultivo de soja. $\mathrm{O}$ índice de diversidade de Shannon-Wiener variou de 0,23 a 0,48 e o de equitabilidade variou de 0,17 a 0,36 .
\end{abstract}

PALAVRAS-CHAVE: Amostragem, diversidade, Hymenoptera, parasitóides, soja.

\section{ABSTRACT}

MINIMUM NUMBER OF MÖRICKE TRAPS IN SAMPLING PARASITIC HYMENOPTERA DIVERSITY IN GLYCINE MAX (L.) MERRILL SOYBEANS. The aim of this study was to evaluate the minimum number of Möricke traps needed to sample the diversity of Hymenoptera parasitoids in Glycine max (L.) Merrill soybeans. The experiment was conducted in a soybean crop of the variety IAC 82, in Ribeirao Preto $\left(21^{\circ} 12^{\prime} 17.8^{\prime \prime} \mathrm{S} / 47^{\circ} 52^{\prime} 34.8^{\prime \prime} \mathrm{W}\right)$, SP, Brazil. In the period from February 24 to April 11, 2003, seven collections of Hymenoptera parasitoids were made with the use of Möricke traps. The statistical design consisted of randomized blocks, with five treatments (one to five traps) and four repetitions. In terms of the total number of families caught, the highest averages were obtained with the use of two to five Möricke traps, these averages differing significantly from the average obtained with one trap. These results suggest that for parcels of 12 x $18 \mathrm{~m}$, two Möricke traps are sufficient to sample the diversity of parasitoids Hymenoptera in soybean crop. The Shannon-Wiener diversity index ranged from 0.23 to 0.48 , and the evenness ranged from 0.17 to 0.36 .

KEY WORDS: Diversity, Hymenoptera, parasitoids, sample, soybean.

\section{INTRODUÇÃO}

A soja (Glycine max (L.) Merrill) é uma leguminosa que chegou ao Brasil com os primeiros imigrantes japoneses em 1908. A partir da década de 1970 observou-se a expansão do seu cultivo e a soja tornou-se um importante produto brasileiro de exportação. No Brasil, na safra 2006/2007, foram cultivados aproximadamente20,6 milhões deha, responsáveis pela produção deaproximadamente58,4 milhões $\operatorname{det}($ EMBRAPA,2008).
A cultura da soja está sujeita ao ataque de insetos praticamente durante todo o seu ciclo; as lagartas Anticarsia gemmatalis Huebner, 1818 e Chrysodeixis includens Walker, 1857 (Lepidoptera, Noctuidae) e os percevejos Euschistus heros (Fabricius, 1794), Nezara viridula (Linnaeus, 1758) e Piezodorus guildinii (Westwood,1837)(Hemiptera, Pentatomidae) são suas principais pragas. Dentre os parasitóides dos lepidópteros da soja, destacam-se o encirtídeo Copidosoma truncatellum (Dalman, 1820), o

${ }^{2}$ Instituto Biológico, Centro Experimental Central, Campinas, SP, Brasil. 
ichneumonídeo Microcharops bimaculata (Ashmead, 1895) e os braconídeos Apanteles marginiventris (Cresson, 1865), Campoletissp. eMeteorusspp. (GAZZONI etal.,1981); como parasitóides deovos de pentatomídeos fitófagos, destacam-se os scelionídeos Trissolcusbasalis (Wollaston, 1858) e Telenomus podisi Ashmead, 1893 (PANizZi; SlansKy Junior, 1985).

No Brasil, são poucos os autores que se dedicam ao desenvolvimento de metodologias de coletas de himenópteros parasitóides. Tais estudos são necessários pois dão suporte metodológico a experimentos que, por exemplo, possibilitem a análise da influência das aplicações de inseticidas e outros agroquímicos na população de himenópteros parasitóides e outros insetos benéficos. O uso de armadilhas de Möricke é amplamente difundidoemestudos delevantamentos faunísticos de himenópteros parasitóides, tanto em ambientes silvestres (Azevedo etal., 2003; Periotoetal., 2003; PeRIOTOetal., 2005), quanto em agroecossistemas (Periotoetal., 2002 a; Periotoetal., 2002b; Periotoetal., 2004; SouZA, 2006). No entanto, não há indicação do número de armadilhas a ser utilizado para a amostragem daqueles agentes de controle. A falta de tal dado pode, por um lado, resultar em subamostragem e, por outro, no aumento desnecessário de seu custo.SÖRENSENet al. (2002) afirmaram que, para programas de monitoramento de longo prazo, o desenho do protocolo deve ser considerado cuidadosamente e propõem que o método de amostragem deve ser prático.

Pouco se conhece a respeito da composição faunística deste grupo de insetos no agroecossistema da soja. Perioto et al. (2000) propuseram o uso de armadilhas de Möricke para a realização de levantamentos faunísticos de himenópteros parasitóides em cultura de soja, dadas as dificuldades apresentadas na utilização de métodos ativos de captura, dentre as quais o pequeno tamanho dos insetos que compõem este grupo. A eficiência do aparato proposto foi comprovada por PERIOTOet al. (2002a) em coletas realizadas com armadilhas de Möricke no Município de Nuporanga, SP, na safra 1999/2000, que capturaram 4.969 himenópteros parasitóides pertencentes a sete superfamílias e 16 famílias; boa parte das espécies coletadas participa do controle natural de diversas pragas daquela leguminosa.

Este estudo teve por objetivos avaliar o número mínimo de armadilhas deMöricke para a amostragem da diversidade de famílias de himenópteros parasitóides em cultura de soja, em Ribeirão Preto,SP.

\section{MATERIAL E MÉTODOS}

O experimento foi conduzido em cultivo de soja, da variedade IAC 82, plantada em 27/12/2002, com
$50 \mathrm{~cm}$ entre linhas em área experimental da Apta RegionalCentro-Leste,SP, $\left(21^{\circ} 12^{\prime} 17,8^{\prime \prime} \mathrm{S} / 47^{\circ} 52^{\prime} 34,8^{\prime \prime} \mathrm{O}\right)$. Utilizou-se o delineamento estatístico de blocos casualizados, com cinco tratamentos e quatro repetições. A variável estudada foi o número de aparatos de coletas, que variou de um a cinco. As parcelas, em número de 20 , mediam 12 × $18 \mathrm{~m}$. Na área experimental foram realizados os tratos culturais recomendados para a região e não foram utilizados métodos de controle de pragas.

No período de 24 de fevereiro a 11 de abril de 2003 (inicio do florescimento até a fase de maturação da cultura) foram realizadas setecoletas dehimenópteros parasitóides com aparatos de coleta adaptados por Perioto et al. (2000). Estes aparatos, compostos por uma armadilha de Möricke (pratos plásticos fundos, amarelos, descartáveis, com $12 \mathrm{~cm}$ de diâmetro, contendo água, formol e detergente), fixada a uma estaca de madeira na altura do terço médio das plantas, permaneceram no campo por 72 horas. Os insetos capturados foram conservados em álcool etílico a $70 \%$ até sua triagem e análise em laboratório. Os parasitóides foram identificados em nível de família segundo FERNANDES; SHARKEY (2006).

Os dados obtidos nas amostragens foram analisados estatisticamente com o programa computacional SAS (2003) pelo procedimento GLM e o modelo utilizado considerou os efeitos fixos de tratamento, bloco e tempo, além das interações duplas entre tais efeitos. Para a análise de diversidade das famílias de himenópteros parasitóides foram aplicados os índices de diversidade $\left(\mathrm{H}^{\prime}\right)$ e de equitabilidade de Shannon-Wiener, para seus cálculos foi utilizado o software Dives (RODRIGUES, 2005).

\section{RESULTADOS E DISCUSSÃO}

Abundância. No presente estudo, foram coletados 21.977 exemplares de himenópteros parasitóides pertencentes a 22 famílias de oito superfamílias (Tabela 1). As famílias mais coletadas foram Encyrtidae (86,9\% do total de himenópteros parasitóides coletados), Scelionidae $(5,3 \%)$, Trichogrammatidae $(3,2 \%)$ e Aphelinidae $(2,1 \%)$. Perioto et al. (2002a) realizaram estudo semelhante em Nuporanga, SP, e relataram a presença de quinze famílias. No entanto, tal diferença é insignificante, dado que o acréscimo de famílias deveu-se ao encontro de exemplares pertencentes a sete famílias com freqüências relativas muito próximas de zero por cento. Cabe ressaltar que as famílias Aphelinidae, Encyrtidae e Trichogrammatidae (Chalcidoidea) e Scelionidae (Platygastroidea) foram as mais abundantes em ambos os estudos, fato que pode ser atribuído à existência de muitos hospe- 
deiros potenciais (lagartas e percevejos) na cultura da soja. A abundância alta de Encyrtidae pode ser creditada a uma espécie do gênero Copidosoma Ratzeburg, que representou $86,8 \%$ do total de himenópteros parasitóides coletados. Trata-se de uma espécie poliembriônica que parasitaC. includens eque, a partir de um ovo depositado pelo parasitóide adulto, ocorre o desenvolvimento, no corpo do hospedeiro, de muitas larvas do parasitóide.

Amostragem. Quanto ao total de famílias capturadas, as maiores médias foram obtidas com a utilização de duas a cinco armadilhas de Möricke, que diferiram significativamente da média obtida com uma armadilha (Tabela 2). Estes resultados indicam que, para parcelas de $12 \times 18 \mathrm{~m}$, duas armadilhas de Möricke são suficientes para amostrar a diversidade de himenópteros parasitóides em cultivo de soja.

As coletas realizadas com duas armadilhas capturaram 4.681 exemplares dehimenópteros parasitóides pertencentesa 17 famílias deHymenoptera,nãoforam capturados exemplares de himenópteros pertencentes àsfamílias Evaniidae (Evanioidea) ePerilampidae e Torymidae (Chalcidoidea). Nas demais coletas estas famílias apresentaram baixa freqüência: os sete exemplares capturados representaram apenas $0,03 \%$ do total de himenópteros parasitóides.
Diversidade. Apesar da grande riqueza de famílias de himenópteros parasitóides (22 famílias), o índice de diversidade $\left(\mathrm{H}^{\prime}\right)$ deShannon-Wiener foi baixoem todos os tratamentos (diferentes números de armadilhas de Möricke) e variou de 0,23 a 0,48 como conseqüência da baixa equitabilidade (J), que variou de 0,17 a 0,36 (Tabela3). Osvalores dediversidadeedeequitabilidade observados devem-se, provavelmente, ao elevado número de exemplares coletados de uma espécie de Copidosoma (Encyrtidade), que representou 86,8\% do total dehimenópteros parasitóides. Nas coletas realizadas com uma armadilha de Möricke, observamos o maior valor de diversidade $(0,48)$ e de uniformidade $(0,36)$ e os encirtídeos representaram $69,8 \%$ do total de himenópteros parasitóides coletados. Nas coletas onde o número de armadilhas variou de dois a cinco, a porcentagem de encirtídeos variou de 84,8 a $89,6 \%$ do total de parasitóides coletados. Tais resultados corroboram a proposição de Odum (1988) o qual afirmou que,em comunidades de regiões tropicais sazonais, poucas espécies são muito abundantes e, portanto, ali dominantes, gerando baixa uniformidade. SegundoHugHes (1986), nas amostras de comunidades a maioria dos indivíduos pertence a um pequeno número de espécies abundantes, enquanto a maioria das espécies é representada por um pequeno número de indivíduos.

Tabela 1 - Total de himenópteros parasitóides coletados por meio de diferentes números de armadilhas de Möricke total e suas freqüências relativas em cultivo de soja Glycine max (L.) Merril, em Ribeirão Preto, SP, 2003.

\begin{tabular}{lrrrrrrrrr}
\hline Superfamílias e famílias & $24 / 2 / 03$ & $8 / 3 / 03$ & $14 / 3 / 03$ & $21 / 3 / 03$ & $28 / 3 / 03$ & $4 / 3 / 03$ & $11 / 3 / 03$ & total & FRS \\
\hline Ceraphronoidea & 1 & 4 & 4 & 6 & 12 & 5 & 6 & 38 & 0,17 \\
Ceraphronidae & 1 & 4 & 4 & 6 & 12 & 5 & 6 & 38 & 0,17 \\
Chalcidoidea & 316 & 338 & 223 & 456 & 13.330 & 4.316 & 1.555 & 2.0534 & 93,43 \\
Aphelinidae & 8 & 19 & 60 & 69 & 166 & 64 & 79 & 465 & 2,12 \\
Chalcididae & 0 & 0 & 0 & 9 & 15 & 2 & 1 & 27 & 0,12 \\
Encyrtidae & 28 & 182 & 52 & 326 & 12.918 & 4.189 & 1.421 & 1.9116 & 86,98 \\
Eulophidae & 6 & 11 & 6 & 1 & 13 & 3 & 7 & 47 & 0,22 \\
Eupelmidae & 0 & 0 & 0 & 3 & 0 & 0 & 0 & 3 & 0,01 \\
Eurytomidae & 0 & 1 & 2 & 0 & 2 & 1 & 0 & 6 & 0,03 \\
Mymaridae & 30 & 10 & 13 & 17 & 33 & 23 & 17 & 143 & 0,65 \\
Perilampidae & 0 & 0 & 0 & 0 & 1 & 0 & 0 & 1 & 0,00 \\
Pteromalidae & 3 & 3 & 4 & 0 & 1 & 0 & 0 & 11 & 0,05 \\
Signiphoridae & 1 & 1 & 0 & 1 & 1 & 0 & 5 & 9 & 0,04 \\
Torymidae & 0 & 0 & 0 & 0 & 1 & 0 & 0 & 1 & 0,00 \\
Trichogrammatidae & 240 & 111 & 86 & 30 & 179 & 34 & 25 & 705 & 3,21 \\
Cynipoidea & 14 & 21 & 16 & 12 & 7 & 2 & 6 & 78 & 0,35 \\
Figitidae (Eucoilinae) & 14 & 21 & 16 & 12 & 7 & 2 & 6 & 78 & 0,35 \\
Chrysidoidea & 1 & 2 & 0 & 2 & 4 & 0 & 0 & 9 & 0,04 \\
Bethylidae & 1 & 2 & 0 & 2 & 4 & 0 & 0 & 9 & 0,04 \\
Evanioidea & 1 & 0 & 0 & 0 & 0 & 1 & 0 & 2 & 0,01 \\
Evaniidae & 1 & 0 & 0 & 0 & 0 & 1 & 0 & 2 & 0,01 \\
Ichneumonoidea & 8 & 13 & 17 & 19 & 19 & 6 & 14 & 96 & 0,44
\end{tabular}

Continua... 
Tabela 1 - Continuação

\begin{tabular}{lcrrrrrrrr}
\hline Superfamílias e famílias & $24 / 2 / 03$ & $8 / 3 / 03$ & $14 / 3 / 03$ & $21 / 3 / 03$ & $28 / 3 / 03$ & $4 / 3 / 03$ & $11 / 3 / 03$ & total & FRS \\
\hline Braconidae & 5 & 6 & 10 & 6 & 9 & 3 & 6 & 45 & 0,20 \\
Ichneumonidae & 3 & 7 & 7 & 13 & 10 & 3 & 8 & 51 & 0,23 \\
Proctotrupoidea & 4 & 0 & 2 & 9 & 2 & 2 & 0 & 19 & 0,09 \\
Diapriidae & 4 & 0 & 2 & 9 & 2 & 2 & 0 & 19 & 0,09 \\
Platygastroidea & 60 & 109 & 321 & 308 & 279 & 73 & 51 & 1.201 & 5,46 \\
Scelionidae & 54 & 105 & 308 & 298 & 275 & 72 & 48 & 1.160 & 5,28 \\
Platygastridae & 6 & 4 & 13 & 10 & 4 & 1 & 3 & 41 & 0,19 \\
\hline Total de parasitóides & 405 & 487 & 583 & 812 & 13.653 & 4.405 & 1.632 & 2.1977 \\
\hline
\end{tabular}

FR= freqüência relativa das famílias e superfamílias de himenópteros parasitóides coletados, em relação ao total de himenópteros parasitóides da superfamília a que pertencem.

Tabela 2 - Número médio de famílias de himenópteros parasitóides coletadas por meio de diferentes números de armadilhas de Möricke em cultivo de soja Glycine max (L.) Merril, em Ribeirão Preto, SP, 2003.

\begin{tabular}{cc}
\hline $\begin{array}{c}\text { oo de armadilhas } \\
\text { de Möricke }\end{array}$ & $\begin{array}{c}\text { Famílias de } \\
\text { himenópteros parasitóides }\end{array}$ \\
\hline 1 & $3,79 \pm 0,26 \mathrm{~b}^{1}$ \\
2 & $5,50 \pm 0,34 \mathrm{ab}$ \\
3 & $6,50 \pm 0,38 \mathrm{a}$ \\
4 & $6,39 \pm 0,28 \mathrm{a}$ \\
5 & $6,71 \pm 0,46 \mathrm{a}$ \\
\hline F & $7,13^{*}$ \\
C.V.(\%) & 11,31 \\
\hline
\end{tabular}

${ }^{1}$ médias \pm erro padrão, seguidas pela mesma letra, não diferem entre si, pelo teste de Tukey, no nível de 5\% de significância.

${ }^{*} \mathrm{p}=0,0035$

\section{CONCLUSÕES}

Nas condições do presente experimento pode-se recomendar o uso de duas armadilhas de Möricke para a amostragem da diversidade de famílias de himenópteros parasitóides na cultura da soja. No entanto, dado que o cultivo desta leguminosa no Brasil é realizado em grande extensão territorial e ocorre sob a influência de fatores edafo-climáticos variados, recomenda-se parcimônia na utilização destes dados, pois é necessária a realização de avaliações semelhantes nas diferentes regiões produtoras para seu estabelecimento como padrão.

\section{AGRADECIMENTOS}

À Dra. Claudia Cristina Paro de Paz, pesquisadora científica da Apta Ribeirão Preto, pela contribuição na análise estatística dos dados.

\section{REFERÊNCIAS}

AZEVEDO, C.O.; CORRÊA, M.S.; GOBBI, F.T.; KAWADA, R.; LANES, G.O.; MOREIRA, A.R.; REDIGHIERI, E.S.; SANTOS, L.M. DOS; WAICHERT, C. Perfil das famílias de vespas parasitóides (Hymenoptera) em uma área de Mata Atlântica da Estação Biológica de Santa Lúcia, Santa Teresa, ES, Brasil. Boletim do Museu de Biologia Mello Leitão (Nova Série), v.16, p.39-56, 2003.

EMBRAPA. Soja em números (safra 2006/2007). Disponível em: <http://www.cnpso.embrapa.br/ index.php? op_page $=294 \&$ cod_pai $=16>$. Acesso em: 14 nov. 2008.

FERNÁNDEZ, F.; SHARKEY, M. (Ed.). Introducción a los Hymenoptera de la Región Neotropical. Bogotá: Sociedad Colombiana de Entomologia y Universidad Nacional de Colombia, 2006. 893p.

GAZZONI, D.; OLIVEIRA, E.B. DE; CORSO, I.C.; FERREIRA, B.S.C.; BÔAS, G.L.V.; MOSCARDI, F.; PANIZZI, A.R. Manejo de pragas da Soja. Londrina: Embrapa CNPSo., 1981. 44p. (Circular Técnica, 5).

HUGHES, R.G. Theories and models of species abundances. American Naturalist, v.128, n.6, p.879-899, 1986.

ODUM, E.P. Ecologia. Rio de Janeiro: Ed. Guanabara Koogan, 1988. 434p.

PANIZZI, A.R.; SLANSKY JUNIOR, F. Review of phytophagous pentatomids (Hemiptera: Pentatomidae) associated with soybean in Americas. Florida Entomologist, v.68, n.1, p.184-214, 1985.

PERIOTO, N.W.; LARA, R.I.R.; SANTOS, J.C.C.; SILVA, T.C. da. Utilização de armadilhas de Moericke em ensaios de seletividade de inseticidas em himenópteros parasitóides. Arquivos do Instituto Biológico, São Paulo, v.67, p.93, 2000. Suplemento. Resumo 133. Trabalho apresentado na rEUNIÃO ANUAL DEO INSTITUTO BIOLÓGICO, 13., 2000, São Paulo. Resumos. 
PERIOTO, N.W.; LARA, R.I.R.; SANTOS, J.C.C.; SILVA, T.C. da. Himenópteros parasitóides (Insecta, Hymenoptera) coletados em cultura de soja (Glycine max (L.)) Merril (Fabaceae), no município de Nuporanga, SP, Brasil. Revista Brasileira de Entomologia, v.46, n.2, p.185-187, 2002a.

PERIOTO, N.W.; LARA, R.I.R.; SANTOS, J.C.C.; SELEGATTO, A. Himenópteros parasitóides (Insecta, Hymenoptera) coletados em cultura de algodão (Gossypium hirsutum L.) (Malvaceae), no município de Ribeirão Preto, SP, Brasil. Revista Brasileira de Entomologia, v.46, n.2, p.165-168, 2002b.

PERIOTO, N.W.; LARA, R.I.R. Himenópteros parasitóides da Mata Atlântica. I. Parque Estadual da Serra do Mar, Ubatuba, SP, Brasil. Arquivos do Instituto Biológico, São Paulo, v.67, n.4, p.441-445, 2003. Disponível em: <http:/ / www.biologico.sp.gov.br/docs/arq/V70_4/perioto.pdf>. Acesso em: 8 abr. 2008.

PERIOTO, N.W.; LARA, R.I.R.; SELEGATTO, A.; LUCIANO, E.S. Himenópteros parasitóides (Insecta, Hymenoptera) coletados em cultura de café Coffea arabica L. (Rubiaceae) em Ribeirão Preto, SP, Brasil. Arquivos do Instituto Biológico, São Paulo, v.71, n.1, p.41-44, 2004. Disponível em: <http://www. biologico.sp.gov.br/docs/arq/ V71_1/perioto2. pdf>. Acesso em: 8 abr.2008.

PERIOTO, N.W.; LARA, R.I.R.; SELEGATTO, A. Himenópteros parasitóides da Mata Atlântica. II.
Núcleo Grajaúna, Rio Verde da Estação Ecológica Juréia-Itatins, Iguape, SP, Brasil. Arquivos do Instituto Biológico, São Paulo, v.72, n.1, p.81-85, 2005. Disponível em meio eletrônico em < http:/ / www.biologico. sp.gov.br/docs/arq/V72_1/perioto2.PDF>. Acesso em: 8 abr. 2008.

RODRIGUES, W.C. DivEs - Diversidade de espécies. Versão 2.0. Software e Guia do Usuário. Disponível em: <http:// www.ebras.vbweb.com.br>. Acesso em: 10 mai. 2005.

SAS/STAT. User's guide: statistics, version 9, v.1, Cary: SAS Institute, NC, 2003.

SÖRENSEN, L.L.; CODDINGTON, J.A.; SHARFF, N. Inventorying and estimating subcanopy spider diversity using semiquantitative sampling methods in an afromontane forest. Environmental Entomology, v.31, n.2, p.319-330, 2002.

SOUZA, L. Composição da fauna de Hymenoptera associada a área agrícola de manejo tradicional: abelhas nativas e parasitóides. 2006. 103p. Tese (Doutorado) - Instituto de Biociências, Universidade Estadual Paulista “Júlio de Mesquita Filho", Rio Claro, 2006.

Recebido em 23/2/07

Aceito em 24/11/08 